\title{
Memoria operativa, ansiedad matemática y habilidad aritmética en docentes de educación básica en formación
}

\author{
Working Memory, math anxiety and arithmetic skills \\ in elementary education preservice teachers
}

\author{
Ismael Esquivel-Gámez ${ }^{1}$ \\ Flora Lilia Barrios-Martínez ${ }^{2}$ \\ Karina Estela Gálvez-Buenfil ${ }^{3}$
}

\begin{abstract}
Resumen: Dado el impacto que tendrán los docentes en formación, en el aprendizaje de las matemáticas por los niños, en cuanto a su actitud hacia las matemáticas y su habilidad aritmética, se ha desarrollado el presente estudio. Para ello, se explora la relación entre los niveles de memoria operativa, ansiedad matemática y habilidad aritmética, en 39 estudiantes de docencia de una institución particular del sureste mexicano. Para medir la memoria operativa en ambos dominios, se usaron tareas de alcance complejo, para la percepción sobre ansiedad matemática se aplicó la escala Perfil de Ansiedad Matemática y para la habilidad aritmética, un conjunto de problemas verbales extraidos de las guías públicas para el examen nacional de ingreso a la educación media superior del Centro Nacional de Evaluación para la Educación Superior en México. Como en otros estudios, existe asociación positiva entre la capacidad de memoria operativa y la habilidad aritmética, y negativa entre la primera y
\end{abstract}

Fecha de recepción: 17 de mayo de 2019. Fecha de aceptación: 25 de octubre de 2019.

1 Universidad Veracruzana, Campus Veracruz, Facultad de Administración, iesquivel@uv.mx, orcid. org/0000-0001-7914-5170.

2 Centro de Actualización del Magisterio en Veracruz, florabarrios@gmail.com, orcid.org/0000-00033729-560X.

${ }^{3}$ Universidad Veracruzana, Campus Boca del Río, Facultad de Pedagogía, karigalfil@hotmail.com, orcid. org/0000-0003-2485-4687. 
el nivel de ansiedad matemática, en su escala de actitudes; lo cual abre posibilidades futuras de mejorar la primera, mediante entrenamiento adaptativo y verificar el impacto en las dos últimas.

Palabras clave: Memoria operativa, ansiedad matemática, habilidad aritmética, docentes en formación

\begin{abstract}
This study has been developed given the impact that will have the attitude towards mathematics and the arithmetic ability of the preservice teachers, with their future students learning math. For this, we explore the relationship between the Working Memory levels, math anxiety and arithmetic skills of 39 students from a private institution on the southeast of Mexico. To measure Working Memory in both domains, complex span task were used, for the math anxiety perception the Mathematical Anxiety Profile scale was applied and for the arithmetic ability, a set of verbal problems extracted from the public guides for the national admission high school exam of the National Center for Evaluation of Higher Education in Mexico. As in other studies, a positive association has been found between the Working Memory capacity and the arithmetic skills, and negative between the first and the level of math anxiety, in its scale of attitudes; which opens future possibilities to improve the first one and verify the impact in the last two.
\end{abstract}

Keywords: Working memory, math anxiety, arithmetic skills, preservice teachers, mathematics education.

\title{
1. INTRODUCCIÓN
}

Investigaciones diversas (Chang y Beilock, 2016; Ruff y Boes, 2014) afirman que las actitudes, emociones y creencias de los profesores impactan de manera importante a la forma en que enfrentan a sus estudiantes en el aula de clases $y$, en especial a las matemáticas ya que pueden transferirles sus miedos y prejuicios al aprendizaje de las mismas; siendo este un factor que influye significativamente en la formación de actitudes positivas o negativas. Los efectos de la ansiedad se pueden manifestar a lo largo de la vida en detrimento del desarrollo vocacional y académico. Tan solo en Estados Unidos 93\% de la población 
adulta ha reconocido haber experimentado cierto grado de AM (Luttenberger, Wimmer, y Paechter, 2018, p.312) y esta situación se replica en muchos otros países donde se han llevado a cabo investigaciones similares. Por lo anterior, se ha desarrollado el presente estudio, cuyo objetivo es medir la capacidad de la memoria operativa, la habilidad aritmética y los índices de ansiedad matemática de estudiantes de docencia y determinar los niveles de asociación entre los constructos. Inicialmente, se documentan los correspondientes constructos, los estudios que han encontrado asociaciones entre ellos, para luego dar paso a la descripción del método y la discusión de resultados, para finalmente presentar las conclusiones.

\section{MARCO TEÓRICO}

\subsection{ANSIEDAD MATEMÁTICA (AM)}

Frecuentemente se define como "sentimientos de tensión y ansiedad que interfieren con la manipulación de los números y la resolución de problemas matemáticos en una amplia variedad de situaciones de la vida y académicas" (Richardson y Suinn, 1972, p. 551). Estos sentimientos negativos suelen desencadenar síntomas físicos como el aumento de la frecuencia cardiaca, sudoración, dolor de cabeza y desorganización mental (Tobias y Weissbrod, 1980, p. 65). Los diversos grados de ansiedad causada por enfrentarse a situaciones que involucran a las matemáticas, bajo condiciones de demanda cognitiva, provoca que se presenten diferencias entre sus habilidades numéricas y desempeño en el área de las matemáticas desde tempranas edades (Vukovic et al., 2013, p. 7), siendo esto un obstáculo a vencer para lograr aprendizajes significativos (García-Santillán et al., 2017, p. 175).

La AM ha sido investigada en los distintos niveles desde la educación básica (Fernández, 2015; Mammarella et al., 2015; Wright, 2017), media superior (Erdem y Keklik, 2013; Mutodi y Ngirande, 2014) y superior (Cleary, 2017; Shi y Liu, 2016a). También se ha estudiado bajo sus distintas dimensiones como la ansiedad estado, ansiedad rasgo (García-López, 2016), ansiedad hacia las evaluaciones (Casari, Anglada, y Daher, 2014), hacia las tareas matemáticas y hacia los cursos de matemáticas o bien en función de las creencias, actitudes y emociones que provoca (Eccius-Wellman, Lara-Barragán, Martschink, y Freitag, 2017). 
Diversos estudios aportan información respecto a los factores que magnifican este problema. Algunos tienen que ver con las características de personalidad del individuo como el temperamento, actitud y motivación propia (Fernández, 2015, p. 29; García-Santillán et al., 2016, p. 369; Rabab’h y Veloo, 2015, p. 4) y otros, con múltiples factores externos como los culturales, sociales y académicos entre otros (Chang y Beilock, 2016, p. 34; Dowker et al., 2016, p. 10). Por su parte, Foley et al. (2017, p. 56), Mutodi y Ngirande (2014, p.289) y Pletzer et al. (2016, p. 12) mencionan que el género también es un factor que puede potenciar a la AM.

Con el fin de medir el grado de ansiedad hacia las matemáticas se han empleado múltiples instrumentos y técnicas a lo largo de más de seis décadas de su estudio. Desde que el concepto de "mathemaphobia" fue acuñado por Gough (1954) han sido creadas y validadas escalas de autopercepción que aportan información relevante al tema: Escala de actitudes hacia las matemáticas (Aiken Jr. y Dreger, 1961, p. 19); MARS (Math Anxiety Rating Scale) de Richardson y Suinn (1972, p. 13); MAS (Mathematics Anxiety Scale) de Fennema y Sherman (1978, p. 325); conformados como la base para diversas adaptaciones al contexto social y cultural de diferentes países.

\subsection{HABILIDAD ARITMÉTICA COMO BASE DE LAS MATEMÁTICAS}

Para la Common Core State Standards Initiative (2019), las matemáticas se conceptualizan en función de seis ramas principales; las cuales incluyen al conocimiento básico de los números (números cardinales, ordinales, conocimiento de los dígitos arábigos y estimación numérica), cálculos numéricos (de uno o más dígitos), fracciones, geometría, álgebra y solución de problemas verbales.

Una habilidad básica que se desarrolla a lo largo del aprendizaje de las matemáticas es la aritmética, que está asociada a la capacidad que desarrollan los estudiantes durante el proceso de resolución de problemas matemáticos básicos, útil en el análisis, razonamiento y proceso de resolución. Esta habilidad es practicada aun en la vida diaria y según Zamora et al. (2018, p. 107) es parte fundamental en el aprendizaje y desempeño de todas las materias relacionadas con la solución de problemas.

Aunio y Räsänen (2016, p. 2) enfatizan el hecho de que las habilidades numéricas desarrolladas en etapas tempranas como la de conteo, aritmética básica, conocimiento de los números, cálculo no verbal, combinación de números, cálculo verbal, comparación lineal y de magnitud, lectura de números y 
relación lógica de los números son predictores de problemas en el futuro desempeño matemático.

En el nivel superior en México, Ramos, Zamora-Lugo y Figueroa-Rodriguez (2018, p. 105) reportan que los resultados del EXANI II (de ingreso al nivel superior) y EXANI III (de ingreso a postgrado) del CENEVAL en el área de pensamiento matemático han decaído entre los años 2014 al 2016, lo que indica deficiencias en los conocimientos básicos para cursar eficientemente dichos niveles educativos.

\subsection{MEMORIA OPERATIVA}

Se encarga de almacenar temporalmente y actualizar una pequeña cantidad de datos, que están accesibles para, mediante el uso de estrategias, manipularlos en tiempo real posteriormente (Jonides, Lacey y Nee, 2005, p. 2). Para operaciones que implican comprender el lenguaje, procesar datos rápidamente y al resolver problemas, preservar los objetivos es de gran importancia (Etchepareborda y Abad-Mas, 2005, p. 80, Flores y Ostrosky-Shejet, 2012, p. 9).

El modelo de componentes múltiples de Baddeley y Hitch de 1974, revisado por Baddeley (2012, p. 6, 2012, p. 12) ha sido especialmente útil para su análisis. Este modelo propone que la memoria operativa está conformada por un componente ejecutivo central, que se divide en dos subsistemas: el bucle fonológico y la agenda visoespacial y, el búfer episódico. La central ejecutiva es una instancia de control de la atención para eventos relacionados con contenidos dentro la MO así como para los que se encuentran fuera de ella (Jaroslawska, Gathercole, Logie y Holmes, 2016, p. 2). El bucle fonológico es el depósito temporal y el repaso de estímulos verbales, que maneja a su vez, dos subsistemas, un almacén de síntesis articulatoria y uno fonológico (Flores y Ostrosky-Shejet, 2012, p. 9). La agenda viso-espacial, mantiene temporalmente información visual y espacial; la cual es usada para planear movimientos y está asociada a la aptitud espacial y tareas vinculadas (Etchepareborda y Abad-Mas, 2005, p. 80). Por su parte, Arteaga y Pimienta (2006, p. 255-259) proponen que está formada por componentes visuales, espaciales y cinéticos. Los primeros encargados de percibir "el qué"; los segundos de la ubicación y transmisión de "el dónde" y los últimos de apoyar el correcto movimiento. Por último, el búfer episódico es el encargado de mantener una relación entre los tres y la memoria de largo alcance; filtrar los estímulos 
conforme a su naturaleza y, aunque es un componente temporal, acceder a la memoria de largo plazo, sea para aprender o recuperar (Saeed, 2011, p. 17-18).

\subsection{CAPACIDAD DE LA MEMORIA OPERATIVA Y SU MEDICIÓN}

De acuerdo con Conway et al. (2005, p. 769), los avances teóricos en el estudio del comportamiento humano desde la revolución cognitiva han colocado a la memoria operativa como un constructo central en la psicología. Para ellos, dado que la $\mathrm{MO}$ es un sistema multicomponente responsable del mantenimiento activo de la información frente al continuo procesamiento y/o la distracción, las tareas de alcance para medir su capacidad, se crearon para requerir no solo el almacenamiento y ensayo, sino también el procesamiento simultáneo de información adicional. Conforme a Carruthers (2016, p. 108), en una tarea de alcance, se maneja un distractor, tal como verificar si una frase es lógica o una ecuación es correcta, mientras se intenta memorizar una lista de elementos no relacionados, por tanto hay dos fuentes de datos: el componente de procesamiento de la tarea y el componente de almacenamiento. Con estos instrumentos y conforme a Kane et al. (2004, p. 774), hay evidencia de que generalmente, quienes recuerdan la mayoría de los estímulos también obtienen mayor precisión en la tarea de procesamiento, por lo cual no existe una compensación entre ambas.

Dado que dichas tareas no son perfectas o puras, ya que las puntuaciones obtenidas están influenciadas por algo estable, con una pequeña contribución de error debido a fluctuaciones aleatorias (Conway et al., 2005, p. 777), una estrategia de investigación es administrar múltiples tareas porque una sola medida contiene la varianza específica de dicha tarea y al recopilarse múltiples medidas, la varianza compartida entre estas tareas es una mejor representación de la CMO. Además, dado que las correlaciones entre tareas de alcance, según Kane et al. (2004, p. 200), generalmente varían de 0.40 a 0.60, sugieren que están aprovechando alguna capacidad común, pero también sugiere que no son idénticas.

A partir del análisis hecho por Conway et al. (2005, p. 783), diversos investigadores han aplicado medidas similares, con la idea de validar sus adecuaciones o bien asociar sus puntajes con los de otros constructos. Enseguida, brevemente se describen estudios previos que han trabajado con poblaciones de universitarios reportados sin desórdenes mentales, como en el presente trabajo y en los cuales se reportan tanto las características de las pruebas como del proceso de aplicación, para tener elementos de constraste con las aquí usadas. 
Debido al tiempo que se consume en aplicar las tradicionales pruebas de alcance, Foster et al. $(2015$, p. 2) probaron un método con 589 universitarios para maximizar la precisión, a la vez que se agilizaba la aplicación de las pruebas de alcance de operaciones, simetría y rotación; cuyas particularidades eran que el número de elementos a recordar y los distractores correspondientes variaba de forma aleatoria de un intento a otro; teniendo tres intentos en cada bloque. Adicionalmente, para reducir la habilidad de las personas para ensayar lo que había que recordar, se les pedía respondieran al distractor (operación, matriz o letra) dentro de un tiempo equivalente a 2.5 desviaciones estándar de su tiempo promedio.

Agarwal, Finley, Rose y Roediger (2016, p. 2) examinaron en 156 universitarios los efectos de la práctica de recuperación en función de: el tiempo transcurrido o el número de elementos entre la práctica inicial y los intentos de recuperación, el retraso entre la práctica inicial y final y la presencia o ausencia de retroalimentación durante la recuperación inicial. La CMO se evaluó de manera individual o grupal con una prueba de alcance de operaciones. Con una duración aproximada de 10 minutos se presentaron 75 pares operación-letra en bloques con tamaños de 3 a 7 pares, presentando diferentes tamaños de manera aleatoria.

Con la intención de examinar las asociaciones combinadas entre el tiempo de reposo y de actividad física con la CMO y el rendimiento académico, Felez-Nobrega et al. (2017, p. 742) trabajaron con 371 universitarios con las pruebas de alcance de operaciones, de simetría y de rotación; aplicadas en grupos de 15 participantes. Las pruebas fueron similares a las propuestas por Foster et al. (2015). Para la prueba de operaciones, se tuvieron dos bloques, cada uno de 25 pares de operación-letra y para las de simetría y rotación, se tuvieron 14 pares, con dos y tres bloques respectivamente.

Felez-Nobrega et al. (2018, p. 2) validaron las pruebas anteriores en una población de 325 universitarios de habla hispana. Para ello, tradujeron las instrucciones del inglés al español y luego de las mediciones, se obtuvieron coeficientes alfa de Cronbach para determinar la consistencia interna y, la validez fue hecha con la comparación de los puntajes de las pruebas con los obtenidos en dos pruebas de razonamiento y un análisis factorial confirmatorio.

El objetivo del estudio de Foster y otros (2017, p. 1678) fue determinar el grupo más beneficiado con entrenamiento cognitivo (baja vs. alta CMO). Para ello, trabajaron con 116 estudiantes entre 18 y 35 años, cuya capacidad se evaluó con pruebas de alcance de rotación y otra de lectura modificada, en la 
cual se tenía que recordar una figura en lugar de una letra. En la primera, se trabajó desde 2 hasta 9 pares letra-flecha y en la segunda, de 3 a 11 pares frase-figura. Ambas pruebas presentaban los estímulos al azar, de modo que no hubo necesidad del contrabalanceo.

Kray y Fehér (2017, p. 4) en su estudio pre y postest, buscaron determinar el impacto de las demandas de inhibición y memoria de trabajo en la transferencia y mantenimiento de mejoras en la conmutación de tareas, inducidas por entrenamiento cognitivo, desde la perspectiva de 81 jóvenes y 81 adultos mayores. Para medir la CMO se usaron las pruebas de alcance de lectura y conteo, recortadas de 12 a 8 intentos y para la actualización de memoria, la prueba Atrás N Verbal $(\mathrm{N}=2,3)$.

Para explorar cómo los estudiantes bilingües utilizan su CMO bajo diferentes cargas cognitivas y entender qué lleva a los bilingües a inhibir una respuesta predominante y permitir una respuesta más adaptable y cuándo y cómo ocurre, fueron los objetivos de Yang y Yang (2017, p. 4). En su comparación, entre 25 estudiantes bilingües y 25 monolingües en inglés como lengua nativa, fueron evaluados en su CMO base, con la prueba de alcance de operaciones en la cual, en una sola pantalla aparecían la operación y la letra a memorizar, todo ello se leía en voz alta y luego bajo el control del participante, se presentaba la siguiente pantalla, hasta terminar.

Yue et al. (2017, p. 6), a partir de estudios que analizan el cerebro como una red en lugar de un conjunto de regiones inconexas, deseaban probar que las redes de alta modularidad favorecían el rendimiento en tareas simples, mientras que las redes de baja, el rendimiento en tareas más complejas. Para ello, midieron la CMO en 52 universitarios con una prueba de alcance de operaciones, la cual manejaba en distintas pantallas, la operación, el resultado y la letra a memorizar. Antes de la aplicación, los estudiantes tuvieron tres bloques de práctica con 2, 3 o 4 ítems. En el primer bloque, solo recordaban la letra, en el segundo resolvían la operación y en el último, ambos.

En su estudio, Li, He, Wang, Hu y Guo (2017, p. 2) investigaron si el entrenamiento en eficiencia de filtrado visual podría mejorar la CMO visual y evaluaron el efecto de transferencia de dicho entrenamiento en la CMO verbal y fluidez de inteligencia, en 38 universitarios diestros con visión normal y sin daltonismo, acreditado con la prueba de Ishihara. Para las mediciones, se usaron las pruebas de alcance de operaciones y palabras, la cual difiere de la primera en que se memoriza una palabra en lugar de una letra. Los participantes leían en voz alta la pareja de elementos verbales y daban su respuesta al presionar uno de dos 
botones. En total, hubo 15 series y 60 parejas operación-palabra, las cuales fueron diferentes pero paralelas en los diversos momentos de medición.

Dokić, Koso-Drljević y Dapo (2018, p. 3) trabajaron con 504 universitarios de primer año, con dos objetivos: verificar el efecto en las propiedades psicométricas de las pruebas de Operaciones, Lectura y Simetría al aplicarse en modalidad grupal; y determinar discrepancias entre aplicar o no, el criterio de precisión mayor al 85\% (Conway et al., 2005, p. 775). Las pruebas se administraron en una sola hora, en orden contrabalanceado, en grupos de dos a cuatro participantes, quienes fueron sentados de modo que cada uno estaba de espalda o de lado respecto de los demás. Se aseguraron que todos siguieran el mismo ritmo, cuidando que comenzaran la tarea al mismo tiempo y que al terminar, permanecieran sentados para evitar distracciones.

Coleman, Watson y Strayer (2018, p. 3) analizaron el cruce factorial de las instrucciones de la tarea (velocidad vs. precisión) y las diferencias individuales en la CMO (alta vs. baja) para producir comparaciones empíricas interesantes que podrían ser teóricamente útiles en esclarecer y disociar diversos mecanismos de control cognitivo. Con ello en mente, hicieron mediciones en 250 universitarias con la prueba de alcance de operaciones, excluyendo aquellas cuya precisión estuvo por debajo del 85\%. Luego se invitó a 50 de ellas, con los puntajes del cuartil más alto (25) y del más bajo (25); para ser evaluadas individualmente con la prueba automatizada de flancos de Eriksen y Eriksen (1974, p. 144); bajo condiciones de velocidad versus precisión.

Es notable encontrar en los estudios revisados, que la prueba de Operaciones es la más común y en ocasiones la única medida de la CMO, lo cual es posible, se deba a lo establecido por Foster et al. (2015, p. 2), sobre la intención de obtener mediciones en menos tiempo, usando pocos instrumentos para obtener conclusiones sobre la $\mathrm{CMO}$. Por lo anterior, en el presente trabajo se ha decidido realizar mediciones con diferentes tareas de la CMO en los dominios visual y verbal, minimizando el tiempo al ser aplicadas de manera grupal y evitando que aqueIlos que son mejores en el procesamiento, aprovechen para ensayar los elementos memorizados, por la forma en que se construyeron las tareas, que se describen más adelante. Las diferencias, con trabajos previos revisados, en cuanto a la funcionalidad de las pruebas usadas y su administración para el presente trabajo, se centran básicamente en que cada prueba tiene 3 intentos en sus 4 niveles, los cuales se distinguen por la cantidad de elementos a memorizar y que van desde 2 hasta 5; con un total de 42 elementos. Cuando aparece el distractor, el participante debe escoger la respuesta de entre dos opciones que 
aparecen en la pantalla. Al momento de recuperar los elementos memorizados, el participante tiene que registrarlos, ya que no cuenta con opciones para elegir. Adicionalmente, una prueba de alcance de razonamiento basada en analogías, adaptada del trabajo de Gutiérrez-Martínez, García-Madruga, Carriedo, Vila y Luzón (2005, p. 8), se modificó para que tuviera una estructura similar a las desarrolladas y validadas por Kane et al. (2004, p. 196). No manejan una sesión de práctica y en su lugar, los participantes pueden, previamente a la resolución, revisar videos demostrativos. Bajo la supervisión de un aplicador, se pueden aplicar, de manera individual o grupal, por lo cual se omite la lectura en voz alta y se resuelven al ritmo del propio participante.

\subsection{MeMORIA Operativa y ANSIEDAd MATEMÁtICA}

Los estudios realizados por Mavilidi, Hoogerheide, y Paas (2014); Sovansky (2013), aportan evidencia significativa del efecto positivo que tiene la disminución de la AM sobre la Capacidad de Memoria Operativa (CMO), potenciándose las habilidades numéricas ya sea por el solo hecho del control de las emociones negativas y pensamientos intrusivos (técnica de la defusión cognitiva) y la implementación de estrategias que la minimicen.

Otras investigaciones (Shi y Liu, 2016a) aportan evidencia del hecho de que al potenciar la CMO se disminuye el nivel de AM y posibilita que la capacidad de razonamiento mejore. Entre los resultados obtenidos por ellos, se menciona el hecho de que sujetos con alta AM manifiestan su condición de estrés principalmente en ejercicios de $\mathrm{MO}$ que involucren a los números como elementos a recordar o manipular y en los niveles de mayor complejidad de las tareas de alcance de lectura. También mencionan que no existe diferencia entre individuos con baja o alta AM en lo que respecta a tiempos de reacción y precisión, para pruebas de $\mathrm{MO}$, que no contengan números.

Múltiples estudios demuestran que existe una correlación negativa entre AM y la CMO (Passolunghi et al., 2016, p. 6; Shi y Liu, 2016b, p. 8; Walker, 2013, p. 44). La relación entre AM y MO ha sido documentada como factor determinante en el pobre desempeño académico en el área de las matemáticas; siendo las mujeres quienes presentan índices de ansiedad significativamente superiores (McAuley, 2015, p. 40). Este último hecho puede deberse a condicionantes externos que las afectan desde muy temprana edad (Geist, 2015, p. 330). 
Ruff y Boes (2014, p. 9) observaron un efecto negativo de la AM sobre el desempeño académico y García-López (2016, p. 36) en su estudio documental sobre la AM describe varias correlaciones existentes con el desempeño académico en el área de las matemáticas, presentándose con la MO como la más fuerte seguida de los estereotipos sociales (género y grupo étnico).

Otros estudios arrojan evidencia significativa de que la CMO del tipo viso-espacial tiene una fuerte influencia sobre la AM, la motivación y los logros matemáticos, además de tener influencia parcial en la relación entre la actitud y los logros matemáticos por lo que sugieren que los maestros deben enfocarse en la mejora de la habilidad viso-espacial para lograr mejoras en el desempeño matemático, ya que los mecanismos mentales para resolver problemas son los mismos desarrollados durante actividades viso-espaciales (Rabab'h y Veloo, 2015, p. 8).

Luttenberger et al. (2018, p.314) concluyen que el desbalance entre AM y $\mathrm{MO}$ se debe a deficiencias en el proceso cognitivo de tal forma que vuelve difícil el resistir las disrupciones de pensamientos irrelevantes que interfieren a los estímulos enfocados a las tareas relevantes. Acorde a Ashcraft y Krause (2007), el bajo desempeño dispara la ansiedad matemática y ésta lleva a bajos desempeños en situaciones relacionadas con tareas numéricas. La AM afecta de manera significativa la fluidez de resolución de problemas matemáticos. Los estudiantes adultos con alta AM son más ineficientes para completar operaciones por minuto con un mayor número de dígitos que, aquellos con baja AM. No solo afecta los procesos cognitivos matemáticos, sino también afecta los procesos cognitivos globales que dependen de la fluidez de inteligencia.

\subsection{MEMORIA OPERATIVA Y HABILIDADES ARITMÉTICAS}

La capacidad de resolver problemas verbales presenta una alta correlación con la MO (Peng et al., 2016, p.457). Los autores concluyen que la correlación entre la MO y las matemáticas en general es de magnitud media $(r=0.35)$ y que los efectos que el tipo de habilidad matemática (conocimiento numérico básico, cálculo numérico, operaciones de un solo dígito, operaciones con varios dígitos, fracciones, resolución de problemas verbales, geometría y álgebra) tiene sobre la relación MO-competencia matemática son significativos. El cálculo numérico y la geometría son las habilidades con mayor y menor correlación con la MO. 
La investigación realizada con estudiantes no graduados por Vallée-Tourangeau, Sirota, y Vallée-Tourangeau $(2016$, p. 3) relacionada con el impacto a la $\mathrm{MO}$ al realizar cálculos mentales con números de un solo dígito sugiere que la interactividad aumenta el grado de recursos de MO y disminuye el efecto de la AM al realizar sumas. Los participantes obtuvieron un mayor porcentaje de respuestas correctas en ausencia de la supresión articulatoria (proceso por el cual hablando se inhibe el desempeño de la memoria, mientras son presentados elementos que hay que recordar) y bajo condiciones de alta interactividad con los elementos.

\section{MÉTODO}

Se partió de un enfoque cuantitativo con un tipo de estudio descriptivo, no experimental, transversal y prospectivo. A continuación, se presentan las características de los participantes, los instrumentos aplicados, el procedimiento seguido y las condiciones de aplicación de las mediciones.

\subsection{PARTICIPANTES}

Formaron parte del estudio, todos los estudiantes $(\mathrm{N}=39)$ de una institución particular formadora de docentes en una zona urbana del centro del estado de Veracruz, México. Los jóvenes formaban parte de las Licenciaturas en Educación Preescolar y Primaria de $1^{\circ}, 3^{\circ}, 5^{\circ}$ y $7^{\circ}$ semestre. La edad de los participantes fue de 18 a 23 años y 97.4\% fueron mujeres. Los participantes leyeron y firmaron un documento dando su consentimiento previo al inicio de la investigación y su participación no estuvo relacionada con asignatura alguna y los resultados obtenidos no afectaron sus calificaciones.

\subsection{INSTRUMENTOS}

Ansiedad Matemática. Se empleó la escala validada para México por Eccius-WeIImann y Lara-Barragán (2016) conocida como "Perfil de Ansiedad Matemática" (PAM). Esta escala de 20 ítems presenta enunciados sobre las actitudes, emociones y creencias alrededor del aprendizaje de las matemáticas. Los ítems 
fueron construidos con base a una escala de Likert desde 1 (casi nunca) hasta 5 (casi siempre) para un mínimo de 20 y un máximo de 100 puntos.

Examen de aritmética. Aunado a la escala de ansiedad, se aplicó un examen electrónico incorporado a una plataforma Moodle, con 12 problemas de aritmética con 4 opciones de respuesta (Ejemplo: Un adolescente envía 42 mensajes por celular en 2 horas. ¿Cuántas horas le tomará enviar 252 mensajes?) Dichos problemas se extrajeron de las guías públicas para el Examen Nacional de Ingreso a la Educación Media Superior (EXANI I) del Centro Nacional de Evaluación para la Educación Superior (CENEVAL, 2018) en México.

Memoria operativa. Se utilizó el software NeuronsWorkout, que consta de ocho tareas automatizadas, seis de ellas de tipo complejo, basadas en el trabajo de Esquivel-Gámez, Balderrama-Trápaga, Vargas-Ortiz y García-Vergara (2018, p. 51). De las seis, cuatro son usadas para el dominio verbal y 2 para el viso-espacial. A continuación, se describen brevemente las citadas pruebas:

Alcance de lectura. Aparecen frases junto con una letra y al tiempo que el participante indica si la frase es lógica o no (ej. El papel se mojó con la tierra cuando lo tiraron), debe memorizar la letra. Cuando termina la serie, se recuperan las letras conforme el orden de aparición.

Alcance de operaciones. Un grupo de pares palabra-operación aritmética aparece, para que el sujeto indique si la operación es correcta o no, al tiempo que memoriza la palabra asociada. Una vez que termina el grupo, las palabras memorizadas se ingresan en el orden en que aparecieron.

Alcance de conteo. Una serie de pares imagen-palabra aparece para que el participante memorice la cuenta de círculos azules e indique si dicha cuenta es un número par o no. Cada imagen contiene círculos y cuadrados azules más círculos verdes. Al final de cada serie, el participante debe registrar las cuentas, conforme al orden en que fueron presentados.

Alcance de razonamiento. Se muestra un conjunto de pares analogía-palabras y el sujeto debe elegir de las palabras, aquella que la complete correctamente. Después de resolver el conjunto, se recuperan las palabras de acuerdo con el orden en que aparecieron.

Alcance de rotación. En una serie aparece una letra en posición normal o girada, luego de indicar SÍ o NO está rotada, desaparece para posteriormente exhibir una flecha que se debe memorizar. Al final de cada serie se debe indicar el orden de presentación de cada flecha. 
Alcance de simetría. En un grupo de pares de matrices, inicialmente aparece una con algunas celdas de negro, de modo que puede presentar simetría desde su eje vertical. El participante deberá marcar sí es o no simétrica, para luego desaparecer y mostrar otra matriz con una celda roja y cuya posición debe memorizarse. Al final de cada grupo, en una matriz vacía se deben registrar, con números consecutivos el orden de aparición de la celda roja.

Para el dominio verbal se tienen a las de alcance de: lectura, operaciones, razonamiento y conteo. Para el viso-espacial, se cuenta con las de alcance de rotación y de simetría.

\subsection{Procedimiento}

Examen de aritmética. El examen se alojó en un curso sobre una plataforma Moodle y para el ingreso a la misma, se concedieron previo a la aplicación, los datos de acceso personales. Se procedió a comunicar el tiempo máximo de resolución (30 mins.), las instrucciones de aplicación del examen y se les prometió un premio monetario a quien obtuviera el mejor resultado. Durante la ejecución, se recordaba frecuentemente a los jóvenes, el tiempo restante y la promesa del premio económico. Al término de la evaluación, se les pidió escribieran en una hoja en blanco sus impresiones de la experiencia

Escala de ansiedad matemática. Al finalizar el examen previo, se indicaron el objetivo y los pasos a seguir, para obtener la percepción de ansiedad matemática, enfatizando que se tomaran su tiempo para responder honestamente a las cuestiones, las cuales estaban integradas en un formulario de Google.

Memoria operativa. Los participantes, organizados en pequeños grupos, se ubicaron en computadoras de modo que dejaran un lugar entre sí, dado que se requería que todos comenzaran cada prueba al unísono y que se redujera la posibilidad de copia. No se ofreció compensación económica alguna y solo se les enfatizó la importancia de la detección temprana de factores personales que pueden impactar en su desempeño académico. 


\section{RESULTADOS}

Una vez concluidas las mediciones, los datos fueron procesados con el software estadístico SPSS V15.0 y la información obtenida se describe enseguida.

Examen de Aritmética. La prueba de normalidad Shapiro-Wilk muestra que, de los resultados obtenidos en la evaluación, solo la edad y calificación total, cumplen tal supuesto. La calificación máxima obtenida fue de 6.54 de una calificación máxima esperada 10 y 84.6\% de los jóvenes obtuvieron una calificación reprobatoria $(<6.0)$. El promedio de calificación obtenido en el examen fue de 3.95 con desviación estándar de 1.27. Dichos resultados parecen indicar un desempeño deficitario en los niveles máximo y promedio, al resolver problemas de aritmética muy básicos. Al buscar asociaciones, se detectó una negativa entre la edad y el tiempo de resolución ( $\mathrm{r}=-0.346$, $\mathrm{p}=0.031$ ) y debido al número tan variable de participantes por grupo, no fue posible llevar a cabo la comparación de medias grupales.

Perfil de Ansiedad Matemática. Al determinar la consistencia interna del instrumento usado, se encontró un Alfa de Cronbach para la escala completa de 0.937 y para sus dimensiones: Actitudes (0.874), Emociones (0.867) y Creencias (0.844). Con los resultados obtenidos, se demuestra una fuerte relación entre las preguntas de cada dimensión, al ser mayores a 0.7 y por tanto, la fiabilidad del instrumento.

De las tres dimensiones, en la correspondiente a actitud, se encontró que, en 5 de sus 8 ítems, $31.6 \%$ de los jóvenes se perciben con mala o pésima actitud ante el hecho de esforzarse en las tareas matemáticas. Para la dimensión de creencias, en cuatro de seis preguntas que la conforman, aproximadamente la mitad (52.6\%) de ellos, se catalogan como poco hábiles o con poca confianza en sus habilidades numéricas. En lo referente a las emociones que generan las matemáticas para los jóvenes, solo 28.9 \% de ellos manifiesta sentirse cómodo con ellas y en la pregunta 15 (Las matemáticas son mi punto fuerte) $55.26 \%$ de los participantes, respondieron casi nunca y $21 \%$ respondieron que a veces, lo que hace que esta pregunta muestre la percepción más baja de los participantes.

Para la escala total se observó que $15.79 \%$ de los participantes manifiesta AM en al menos $75 \%$ de las situaciones contempladas en los 20 ítems, así como otro $13.16 \%$ presenta condiciones de ansiedad entre 50 y $75 \%$ de las ocasiones presentadas en la escala. $65.75 \%$ señala experimentar ansiedad en, cuando mucho, la mitad de las circunstancias planteadas y solo en $5.3 \%$ de los 
casos, manifestaron no experimentar ansiedad bajo alguna de las situaciones presentadas en los ítems.

Memoria operativa. En cada nivel de prueba, se obtienen los datos: respuestas correctas, respuestas en orden, el tiempo promedio de reacción a cada planteamiento y el porcentaje de precisión ante el cuestionamiento distractor. Para obtener los puntajes de las pruebas, en línea con Conway et al. (2005, p. 776), se usó el procedimiento de puntuación de carga unitaria (PCU). Por cada prueba, los estadísticos descriptivos de tales puntajes junto con el tiempo de reacción y porcentaje de precisión, así como el alfa de Cronbach, se muestran en la Tabla 1. En todas las pruebas, se encontró un nivel de asociación medio altamente significativo (desde 0.409 hasta 0.670) entre el PCU y la precisión en las respuestas al distractor y aunque no significativo, se obtuvo un nivel bajo de asociación negativa entre la precisión y el tiempo de reacción, excepto para la prueba de simetría.

Tabla 1. Estadísticos descriptivos

\begin{tabular}{lccccccc}
\hline & Puntaje (PCU) & \multicolumn{2}{c}{ Precisión } & \multicolumn{2}{c}{$\begin{array}{c}\text { Tiempo de } \\
\text { reacción }\end{array}$} & $\begin{array}{c}\text { Consistencia } \\
\text { Interna }\end{array}$ \\
\hline Prueba & Media & $\begin{array}{c}\text { Desv. } \\
\text { típica }\end{array}$ & Media & $\begin{array}{c}\text { Desv. } \\
\text { típica }\end{array}$ & Media & $\begin{array}{c}\text { Desv. } \\
\text { típica }\end{array}$ & $\begin{array}{c}\text { Alfa de } \\
\text { Cronbach }\end{array}$ \\
\hline Conteo & 0.889 & 0.129 & 95.43 & 7.299 & 3.82 & 1.114 & .818 \\
\hline Lectura & 0.896 & 0.098 & 91.05 & 8.689 & 7.26 & 3.497 & .684 \\
\hline Operaciones & 0.876 & 0.110 & 79.85 & 13.800 & 5.84 & 2.804 & .587 \\
\hline Razonamiento & 0.815 & 0.139 & 93.51 & 5.454 & 7.09 & 1.915 & .772 \\
\hline Rotación & 0.635 & 0.170 & 96.34 & 8.953 & 6.07 & 2.865 & .698 \\
\hline Simetría & 0.705 & 0.175 & 81.85 & 13.013 & 6.46 & 2.351 & .778 \\
\hline
\end{tabular}

A partir de la revisión del criterio de la distribución normal de los puntajes (PCU) se aplicaron las pruebas estadísticas correspondientes, encontrando que al comparar los promedios de los puntajes por dominio, las de naturaleza verbal tuvieron una ventaja altamente significativa contra la del tipo viso-espacial (0.86 vs. 
0.67). Aunque el tamaño de la muestra es relativamente pequeño, se calculó el nivel de consistencia interna resultando igual a 0.900 en lo global y por prueba, niveles aceptables.

Digno de comentar, es que, para el caso de la prueba de Operaciones, la cual se caracteriza por tener a la resolución de operaciones aritméticas básicas como elemento distractor, se obtuvo: el promedio menor en la precisión, la mayor dispersión de la misma, un nivel mediano de asociación altamente significativo $(\mathrm{r}=0.570)$ del tiempo de reacción con la precisión y uno más bajo con el PCU ( $r=0.306, p=0.05)$, la única diferencia significativa entre grupos en el PCU $\left(\mathrm{Chi}^{2}=8.664, \mathrm{p}=0.034\right)$, desfavorable para el grupo de séptimo semestre y finalmente, una correlación elemento-total negativa de dicho puntaje en su nivel cuatro, que al eliminarse, resultaría un nivel de consistencia interna de 0.810 .

Asociación entre puntajes de las pruebas. En la Tabla 2 se presentan los niveles de correlación y significancia para las pruebas de Memoria Operativa, en la cual digno de comentarse es el nivel de asociación, altamente significativo, entre la prueba de simetría y tiempo de reacción, posiblemente debido a que se usa un patrón similar tanto en la etapa de procesamiento como en la memorización. Quizás por lo mismo, fue la prueba de tipo viso-espacial que obtuvo un nivel asociativo mayor que una verbal (Lectura), entre el puntaje y la precisión. Adicionalmente, se encontró correlación negativa y significativa entre la edad de los participantes y los puntajes de las pruebas de lectura y de razonamiento $(-0.352$ y -0.319 respectivamente), las cuales requieren de una lectura comprensiva de frases. 
Tabla 2. Asociación entre puntajes de pruebas de MO

\begin{tabular}{|c|c|c|c|c|c|c|c|}
\hline & Rotación & Operaciones & Simetría & $\begin{array}{l}\text { Razona- } \\
\text { miento }\end{array}$ & Conteo & $\begin{array}{l}\text { Tiempo de } \\
\text { reacción }\end{array}$ & Precisión \\
\hline Lectura & $.475\left(^{* *}\right)$ & $\left..482^{* *}\right)$ & $.382\left({ }^{*}\right)$ & $\left..4533^{* *}\right)$ & $.351(*)$ & .031 & $\left..435^{(* *}\right)$ \\
\hline Rotación & 1 & $.478\left(^{* *}\right)$ & $.696^{(* *)}$ & $.458\left(^{* *}\right)$ & .304 & .033 & $.409\left(^{*}\right)$ \\
\hline Operaciones & & 1 & $.561\left(^{* *}\right)$ & $\left..419{ }^{* *}\right)$ & $.406(*)$ & .306 & $\left..505^{* *}\right)$ \\
\hline Simetría & & & 1 & $.447\left(^{* *}\right)$ & $.426(* *)$ & $.467\left({ }^{* *}\right)$ & $.477\left(^{* *}\right)$ \\
\hline Razonamiento & & & & 1 & $.339\left(^{*}\right)$ & -.079 & $\left..670^{* *}\right)$ \\
\hline Conteo & & & & & 1 & .096 & $.482(* *)$ \\
\hline
\end{tabular}

${ }^{*} \mathrm{p}<.05,{ }^{* *} \mathrm{p}<.01$.

Al buscarse la asociación entre la $\mathrm{AM}$ y la $\mathrm{MO}$, se encontraron en sentido negativo y de magnitud moderada unas entre el puntaje de la prueba de razonamiento y la percepción total de la AM ( $\mathrm{r}=-0.391, \mathrm{p}=0.018)$ y otras, con la dimensión de Actitudes $(r=-0.435, p=0.008)$ y de Creencias $(r=-0.420, p=0.011)$. De manera análoga, con las medias globales por dominio, se encontró una de magnitud moderada entre la dimensión Actitudes y el dominio verbal $(r=-0.381, p=0.022)$ y con la AM global, no se encontró asociación significativa alguna.

Entre la calificación obtenida en el examen de aritmética y el nivel de AM, no se encontró asociación alguna, ni en lo global ni por escala. Sin embargo, entre dicha calificación y los puntajes globales de la MO por dominio, se encontró una positiva de magnitud moderada, tanto con lo viso-espacial $(r=0.313$, $\mathrm{p}=0.053)$ como con lo verbal $(\mathrm{r}=0.443, \mathrm{p}=0.005)$, particularmente con la prueba de Lectura $(r=0.418, p=0.007)$.

\section{DISCUSIÓN DE RESULTADOS}

Examen de Aritmética. En función de los resultados, al analizar los ítems, para el índice de facilidad, se encontraron: una pregunta fácil, una bastante fácil, cuatro adecuadas al nivel del estudiante promedio, tres moderadamente difíciles, una difícil y dos muy difíciles. Solo las preguntas muy difíciles arrojan resultados insatisfactorios de desviación estándar (inferiores al 33\%). La ponderación efectiva de la mitad de los reactivos indica que tienen un mayor efecto que el 
deseado en la dispersión de las calificaciones (preguntas 2, 3, 7, 9, 11 y 12). Ahora bien, de los 12 ítems, 7 muestran un índice de discriminación cero o negativo y solo la pregunta 3 obtiene un nivel adecuado (37.54\%), indicando que es efectiva para discernir entre los participantes más de los menos capaces. En cuanto al análisis de la eficiencia descriminativa, los mismos ítems obtuvieron un valor cero o negativo, señalando que preguntan algo diferente al resto o son preguntas erróneas. Al parecer dichos ítems deberían descartarse, sin embargo, dado que han sido analizados a partir de resultados de poblaciones más amplias, de manera que forman parte de la guía del CENEVAL, se considera que tanto el índice como la eficiencia discriminativa, se hayan visto afectados por el bajo desempeño del grupo evaluado.

Además, de la revisión de los comentarios que de manera anónima escribieron al término del examen, digno de comentar es: Solo dos participantes expresaron gusto por las matemáticas, mientras que el resto indicó entre otras cosas que, no recordaban o no sabían como resolver los planteamientos, se sentían estresadas o nerviosas y un rechazo manifiesto a las matemáticas. Si a ello se agrega que en algunos sistemas de educación superior, un puntaje menor a 7 es reprobatorio, todos los participantes (38 mujeres y 1 hombre) estarían reprobados, sin importar el tiempo transcurrido de haber tenido el útimo contacto con la aritmética. Lo anterior puede estar acorde a lo descrito por Peng et al. (2016, p. 461), de que la habilidad de cálculo numérico de los docentes de educación básica en formación en general es deficiente, lo que posiblemente impacta a la MO y al desempeño en la evaluación. Si además se considera que los estudiantes próximos a egresar, obtuvieron el puntaje más bajo, la situación que enfrentarán los docentes en formación y los niños bajo su cuidado, es aún más preocupante.

Perfil de Ansiedad Matemática. Es interesante observar que los ítems sobre creencias y actitudes impactan en mayor proporción al factor de ansiedad de los maestros en formación del presente estudio coincidiendo con los hallazgos de Eccius-Wellman et al. (2017, p.78-81).

Usando el mismo instrumento y aun cuando trabajaron con una muestra mayor ( $\mathrm{n}=285$ ) en un entorno educativo similar, para Casis et al. (2017, p. 188199), sus estudiados indicaron menores niveles de ansiedad media en lo referente a las emociones que los del presente estudio (3.51 vs. 1.75). Una condición similar se tuvo en la confianza en sus habilidades (3.27 vs. 3.18) y en que las matemáticas los hacen sentir incómodos y confusos (2.27 vs. 2.93). En lo referente a creencias, los estudiantes chilenos (Casis et al., 2017) piensan que pueden obtener mejores resultados que sus pares mexicanos (2.92 vs. 2.44). 
Así mismo, 15.79\% de las participantes en el estudio manifiestan rasgos de alta AM; comparándolos con 17\% descrito por Luttenberger, Wimmer, y Paechter (2018, p. 312) de la población americana en general con la misma condición. En línea con ellos, se ha detectado que a mayor edad y en especial las mujeres con alta AM, se perciben como menos hábiles en tareas que incluyan a las matemáticas y creen tener un bajo desempeño durante los exámenes.

En este orden de ideas, Ramírez, Shaw, y Maloney (2018) reportan que más de $80 \%$ de los estudiantes universitarios presentan grados de moderados a altos de AM contra $94.7 \%$ observado en esta investigación.

Memoria Operativa. Los niveles de asociación encontrados entre el puntaje de memoria operativa por prueba y el porcentaje de precisión, implica que aquellos participantes con los mejores resultados en la tarea de manejo de distractores alcanzaron puntuaciones de MO más altas, en línea con Conway et al. (2005, p. 774). Por este motivo, es recomendable alentar a los participantes de estudios similares a que se enfoquen por igual en la memorización y en el procesamiento de la tarea. En cuanto a la media de los puntajes, las menores se dan en las pruebas viso-espaciales, posiblemente debido a que las tareas que manejan estímulos verbales tienen que ver con habilidades más maduras como son el conteo, la aritmética y la lectura y, por tal razón sus puntajes son mayores. Además, el menor tiempo de reacción promedio, se obtiene en la prueba de conteo, que consiste simplemente en contar un tipo de figura. Por otro lado, la media más alta en el PCU se obtiene en la prueba de lectura, posiblemente porque solo es necesario memorizar una letra, así como en el tiempo de respuesta, dado que es necesario procesar frases más extensas. En cuanto a la precisión, posiblemente porque solo es necesario revisar si una letra está o no rotada, se obtuvo la mayor media en la prueba de rotación.

El nivel de asociación entre las diferentes mediciones de la MO va en línea con los resultados de Kane et al. (2004, p. 200), excepto para la tarea de conteo, cuyo nivel fue menor a 0.40 con otras tres; posiblemente debido a la exigencia de traducir un estímulo visual (círculos azules) a uno verbal por recordar (número).

\subsection{ASOCIACIÓN ENTRE PUNTAJES}

De manera particular y en contraste con Peng et al. (2016, p. 457), la solución de problemas verbales del examen de aritmética, presenta una moderada correlación con la capacidad de memoria operativa en ambos dominios, siendo 
altamente significativa para lo verbal, en particular con la prueba de alcance de lectura. Al resolver los problemas, que en promedio manejaban 27 palabras, se requería de una lectura comprensiva y dado que su nivel de desempeño se ha encontrado asociado con la CMO verbal (Esquivel, Martínez, Córdoba y Reyes, 2016, p. 49), de ahí la calificación obtenida. A lo anterior puede añadirse, la asociación encontrada entre el porcentaje de precisión en la prueba de operaciones y su tiempo de respuesta tan bajo, lo cual podría estar asociado a una actitud de negación ante las matemáticas, situación que pudo palparse en los comentarios escritos al final de la prueba.

De los resultados obtenidos por Novak y Tassell (2017, p. 21) en una universidad pública al sureste central de los Estados Unidos ( $\mathrm{n}=47$ ), al buscar asociaciones entre el puntaje ante problemas verbales de matemáticas y la $\mathrm{MO}$ (prueba de alcance de operaciones) se encontró una asociación de nivel similar ( $r=0.18)$ e igualmente no significativa. Al comparar la asociación entre dicho puntaje y el de una similar a la prueba de alcance de rotación, manifiestan una correlación muy baja y no significativa, a diferencia de la aquí encontrada $(r=0.478)$.

La correlación encontrada entre la AM y la MO (prueba de alcance de razonamiento) fue en grado moderado a diferencia de los resultados reportados por Walker (2013, p. 65) donde reportan una relación de mayor magnitud también negativa ( $\mathrm{r}=-0.640)$; sin embargo, los instrumentos de medición fueron totalmente distintos.

Según Rabab'h y Veloo (2015, p. 8) la habilidad viso-espacial es mediadora entre la actitud hacia las matemáticas y el logro matemático ( $r=0.648)$, entre la motivación y el desempeño ( $\mathrm{r}=0.648$ ) y entre la AM y el logro (0.640), lo que motiva a los estudiantes a aprender matemáticas y permite que la actitud ante ellas mejore. Para el presente caso, no se encontró dicha mediación, ya que al introducir el puntaje promedio de las pruebas viso-espaciales, al modelo de regresión lineal para cada una de las escalas de la AM, teniendo a la calificación de aritmética como variable dependiente, no se obtuvieron resultados favorables.

\section{CONCLUSIONES}

Para la población estudiada, sus creencias y actitudes han impactado de mayor manera al nivel de ansiedad percibido, en línea con Eccius-Wellman et al. (2017). Lo anterior posiblemente, pudo conducir a un nivel deficiente en la prueba de 
aritmética, sobre todo si se considera que resolvieron problemas básicos, lo cual a su vez pudo disparar la AM.

Conforme a Ashcraft y Krause (2007) y Sokolowski, Hawes y Lyons (2019), la ansiedad matemática conduce a un patrón de evitación global: los estudiantes evitarán tomar cursos de matemáticas y situaciones en las que las matemáticas sean necesarias, incluidas las carreras profesionales; siempre que sea posible. Para el presente caso, dado el tamaño tan reducido de la población estudiada, no es posible generalizar los resultados hacia la elección de estudios universitarios y mucho menos a la decisión de estudiar docencia.

Sin embargo, la habilidad y rechazo mostrados, podría afectar su desempeño en las evaluaciones previstas para el ingreso al servicio magisterial. Con ello, se estaría en línea con la tendencia a la baja, encontrada por Ramos, Zamora-Lugo y Figueroa-Rodriguez (2018, p. 105) en el área de pensamiento matemático del EXANI III de CENEVAL. Por lo anterior, es necesario tanto fortalecer los conocimientos y habilidades matemáticas, así como desarrollar actitudes y creencias positivas en los maestros en formación, que a su vez permitan desarrollarlas en sus futuros estudiantes.

Aunado a lo anterior y conforme a Ball, Thames y Phelps (2008, p. 405), dada la naturaleza de la enseñanza de las matemáticas, es necesario determinar cuáles aspectos del conocimiento del contenido por enseñar, predicen mejor el desempeño estudiantil, para entonces analizar distintas propuestas para el desarrollo de los profesores en formación que mejoren dicho conocimiento, mediante la implementación de materiales que lo apoyen tanto en su etapa aprendiz como en su etapa profesional.

Aunque con diferentes instrumentos de medición y tamaños de muestra, el hecho de encontrar valores de correlación menores entre AM y MO respecto a otros estudios puede deberse al hecho de que los jóvenes participantes del presente estudio, no se verían afectados en ninguna materia del semestre por un pobre desempeño en el examen y la promesa de un premio monetario no fue suficiente para motivarlos a esforzarse. Por tanto, se requiere implementar mediciones adicionales en muestras similares de estudiantes que permitan, al tiempo de mejorar los procedimientos de medición, determinar con mayor certeza los hallazgos, sobre todo porque en la muestra estudiada, la asociación entre el nivel de ansiedad, global y por escala, con el desempeño aritmético, no ha sido significativa. Y, aunque el nivel encontrado en la habilidad aritmética es preocupante, se hace necesario ampliar el estudio a otros dominios de resolución de problemas matemáticos (geometría y problemas no verbales, entre otros); 
además de verificar sus niveles de asociación con el dominio verbal y el viso-espacial de la memoria operativa.

Conforme a los niveles de asociación encontrados, se abre la oportunidad de implementar un ambiente virtual para docentes en formación, en el cual convivan tanto mediciones automatizadas como tareas adaptativas de entrenamiento (aumento de la dificultad a partir del desempeño) de la memoria operativa; aunadas a tutoriales de aritmética con instrumentos de evaluación formativa, que permitan en los estudiantes, logros que mejoren su confianza. Si además, se usan instrumentos de reconocimiento facial mientras resuelven exámenes de aritmética, se estará ante la posibilidad de enriquecer las mediciones de la ansiedad ante los exámenes, las cuales se basan en gran medida en cuestionarios de autopercepción. Con ello, se podrá proporcionar evaluaciones válidas y útiles para los profesionales psicoeducativos, interesados en detectar aquellos estudiantes con riesgo de deserción y en potenciar sus habilidades y conocimientos matemáticos.

\section{REFERENCIAS}

Aiken Jr., L. R., y Dreger, R. M. (1961). The effect of attitudes on performance in mathematics. Journal of Educational Psychology, 52(1), 19-24. http://doi.org/10.1037/ h0041309

Agarwal, P. K., Finley, J. R., Rose, N. S., and Roediger III, H. L. (2017). Benefits from retrieval practice are greater for students with lower working memory capacity. Memory, 25(6), 764-771.

Arteaga, G. y Pimienta, H. (2006). Memoria operativa y circuitos corticales, Revista de la Facultad de Medicina Universidad Nacional Colombia, 54(4), pp. 248-268. https://doi. org/10.15446/revfacmed

Ashcraft, M. H., y Krause, J. A. (2007). Working memory, math performance, and math anxiety. Psychonomic bulletin and review, 14(2), 243-248.

Aunio, P., y Räsänen, P. (2016). Core numerical skills for learning mathematics in children aged five to eight years -a working model for educators. European Early Childhood Education Research Journal, 24(5), 684-704. https://doi.org/10.1080/1350293X.2014.996424

Baddeley, A. (1983). Working Memory. Philosophical Transactions of the Royal Society of London B: Biological Sciences, 302(1110), 311-324. https://doi.org/10.1098/ rstb.1983.0057 
Baddeley, Alan (2012). Working memory: theories, models, and controversies, Annual Review of Psychology, 63, 1-29.

https://doi.org/10.1146/annurev-psych-120710-100422.

Ball, D.L., Thames, M. H., and Phelps, G. (2008). Content knowledge for teaching: What makes it special? Journal of Teacher Education, 59(5), 389-407.

Blazer, C. (2011). Strategies for Reducing Math Anxiety. Information Capsule. Volume 1102. http://eric.ed.gov/?q=math+anxiety+technology+education \&ft=on\&ff1=dtySince_2009\&id=ED536509

Carruthers, P. (2016). La evolución de la memoria de trabajo, Ludus Vitalis, 40(21), 99-124. http://www.centrolombardo.edu.mx/ludus-vitalis/la-maquinaria-mental-humana-num-40-2013/la-evolucion-de-la-memoria-de-trabajo-ludus-vitalis/

Casari, L. M., Anglada, J., y Daher, C. (2014). Estrategias de afrontamiento y ansiedad ante exámenes en estudiantes universitarios. Revista de Psicología (PUCP), 32(2), 243-269.

Casis, M., Castro, N. R., y Martínez, E. C. (2017). Motivación, autoconfianza y ansiedad como descriptores de la actitud hacia las matemáticas de los futuros profesores de educación básica de Chile. PNA, 11(3), 181-203.

Casoetto, S. D. (2014). Ansiedad rasgo y ansiedad matemática en el ámbito de la educación superior: correlaciones y diferencias de género (UADE). http://repositorio.uade. edu. ar/xmlui / bitstream/handle/123456789/2475/Casoet to. pdf?sequence=1反isAllowed $=y$

CENEVAL. (2018). Exámenes Nacionales de Ingreso (EXANI) - Ceneval. http://www.ceneval.edu.mx/examenes-nacionales-de-ingreso-exani-

Chang, H., y Beilock, S. L. (2016). The math anxiety-math performance link and its relation to individual and environmental factors: a review of current behavioral and psychophysiological research. Current Opinion in Behavioral Sciences, 10, 33-38. http://doi. org/10.1016/j.cobeha.2016.04.011

Cleary, L. (2017). Investigating the Impact of a Brief Cognitive Defusion Intervention on State Anxiety and Psychological Inflexibility/Avoidance (Phd, National University of Ireland Maynooth). http://eprints.maynoothuniversity.ie/8751/\}

Coleman, J. R., Watson, J. M., and Strayer, D. L. (2018). Working memory capacity and task goals modulate error-related ERPs. Psychophysiology, 55(3), e12805.

Conway, A. R., Kane, M. J., Bunting, M. F., Hambrick, D. Z., Wilhelm, O., y Engle, R. W. (2005). Working memory span tasks: A methodological review and user's guide. Psychonomic Bulletin Review, 12, 769-786. http://link.springer.com/article/10.3758/BF03196772

Đokić, R., Koso-Drljević, M., and Đapo, N. (2018). Working memory span tasks: Group administration and omitting accuracy criterion do not change metric characteristics. PloS one, 13(10), e0205169. 
Dowker, A., Sarkar, A., y Looi, C. Y. (2016). Mathematics Anxiety: What Have We Learned in 60 Years? Frontiers in Psychology, 7. https://doi.org/10.3389/fpsyg.2016.00508

Eccius-Wellman, C., Lara-Barragán, A. G., Martschink, B., y Freitag, S. (2017). Comparación de perfiles de ansiedad matemática entre estudiantes mexicanos y estudiantes alemanes. Universia, VIII(23), 69-83. https://doi.org/10.22201/iisue.20072872e.2017.23.3011

Eccius-Wellmann, C.-C., y Lara-Barragán, A. G. (2016). Hacia un perfil de ansiedad matemática en estudiantes de nivel superior. Revista Iberoamericana de Educación Superior, 7(18), 109-129.

Erdem Keklik, D., y Keklik, İ. (2013). Motivation and Learning Strategies as Predictors of High School Students' Math Achievement. Cukurova University Faculty of Education Journal, 42(1), 96-109.

Esquivel, I., Martínez, W., Córdoba, R. y Reyes, C. (2016). Memoria operativa y lectura comprensiva: medición con pruebas de amplitud lectora y tipo cloze en ámbitos pre y universitarios. Apertura, 8(2). 38-53. https://dx.doi.org/10.18381/Ap.v8n2.919

Esquivel-Gámez, I., Balderrama-Trápaga, J. A., Vargas-Ortiz, M. C. y García-Vergara, N. (2018). La capacidad de la memoria operativa y su medición automatizada. En I. Esquivel-Gámez, G. Aguirre, R. Edel y J. Balderrama (Coordinadores). Memoria operativa: medición y propuesta para su desarrollo, apoyadas en TIC. Editorial Porrúa Print.

Eriksen, B. A., and Eriksen, C. W. (1974). Effects of noise letters upon the identification of a target letter in a nonsearch task. Perception and Psychophysics, 16(1), 143-149.

Etchepareborda, M.C. y Abad-Mas, L. (2005). Memoria de trabajo en los procesos básicos del aprendizaje, Revista de Neurología, 40(1), 79-83.

Fennema, E., y Sherman, J. A. (1978). Fennema-Sherman Mathematics Attitudes Scales: Instruments designed to measure attitudes toward the learning of mathematics by females and males. Journal for Research in Mathematics Education, 7, 324-326. https://doi.org/10.2307/748467

Fernández Cueli, M. (2015). Análisis de la eficacia de formatos digitales para la enseñanza y aprendizaje de las matemáticas en tercer ciclo de educación primaria. http:// digibuo.uniovi.es/dspace/handle/10651/33296

Flores, J. y Ostrosky-Shejet, F. (2012). Desarrollo neuropsicológico de lóbulos frontales y funciones ejecutivas, Manual Moderno.

Foley, A. E., Herts, J. B., Borgonovi, F., Guerriero, S., Levine, S. C., y Beilock, S. L. (2017). The Math Anxiety-Performance Link: A Global Phenomenon. Current Directions in Psychological Science, 26(1), 52-58. https://doi.org/10.1177/0963721416672463

Foster, J. L., Harrison, T. L., Hicks, K. L., Draheim, C., Redick, T. S., and Engle, R. W. (2017). Do the effects of working memory training depend on baseline ability level? Journal of Experimental Psychology: Learning, Memory, and Cognition, 43(11), 1677. 
Foster, J. L., Shipstead, Z., Harrison, T. L., Hicks, K. L., Redick, T. S., nd Engle, R. W. (2015). Shortened complex span tasks can reliably measure working memory capacity. Memory and cognition, 43(2), 226-236.

Felez-Nobrega, M., Foster, J. L., Puig-Ribera, A., Draheim, C., and Hillman, C. H. (2018). Measuring working memory in the Spanish population: Validation of a multiple shortened complex span task. Psychological Assessment, 30(2), 274.

Felez-Nobrega, M., Hillman, C. H., Cirera, E., and Puig-Ribera, A. (2017). The association of context-specific sitting time and physical activity intensity to working memory capacity and academic achievement in young adults. The European Journal of Public Health, 27(4), 741-746.

García-López, A. (2016). Ansiedad a las matemáticas. http://tauja.ujaen.es/jspui/ handle/10953.1/4092

García-Santillán, A., Escalera-Chávez, M. E., Moreno-García, E., y Santana-Villegas, J. del C. (2016). Factors that Explains Student Anxiety toward Mathematics. Eurasia Journal of Mathematics, Science and Technology Education, 12(2), 361-372. https://doi. org/10.12973/eurasia.2016.1216a

García-Santillán, A. G., Jutta Schnell, J., y Ramos-Hernández, J. R. (2017). Factores que determinan el nivel de ansiedad hacia la matemática en alumnos de nivel superior. Pensamiento Matemático, 7(1), 165-179.

Geist, E. (2015, Spring). Math Anxiety and the "Math Gap": How Attitudes Toward Mathematics Disadvantages Students as Early as Preschool [Text]. http://www.ingentaconnect.com/content/prin/ed/2015/00000135/00000003/art00009

Gough, O. P. S. M. F. (1954). Why Failures in Mathematics? Mathemaphobia: Causes and Treatments. The Clearing House: A Journal of Educational Strategies, Issues and Ideas, 28(5), 290-294. https://doi.org/10.1080/00098655.1954.11476830

Gutiérrez-Martínez, F., y Ramos, M. (2014). La memoria operativa como capacidad predictora del rendimiento escolar. Estudio de adaptación de una medida de memoria operativa para niños y adolescentes. Psicología Educativa, 20(1), 1-10. https://doi. org/10.1016/j.pse.2014.05.001

Jonides, J.; Lacey, S. C. y Nee, D. E. (2005). "Processes of working memory in mind and brain", en Current Directions in Psychological Science, 14(1), 2-5. https://doi. org/10.1111/j.0963-7214.2005.00323.x

Hamid, M. H. S., Shahrill, M., Matzin, R., Mahalle, S., y Mundia, L. (2013). Barriers to Mathematics Achievement in Brunei Secondary School Students: Insights into the Roles of Mathematics Anxiety, Self-Esteem, Proactive Coping, and Test Stress. International Education Studies, 6(11), 1-14. 
Hill, B. D., Foster, J. D., Sofko, C., Elliott, E. M., y Shelton, J. T. (2016). The interaction of ability and motivation: Average working memory is required for Need for Cognition to positively benefit intelligence and the effect increases with ability. Personality and Individual Differences, 98, 225-228. https://doi.org/10.1016/j.paid.2016.04.043

Home I Common Core State Standards Initiative. (2019). 7 de marzo de 2019, http://www. corestandards.org/

Jaroslawska, A., Gathercole, S., Logie, M., y Holmes, J. (2016). Following instructions in a virtual school: Does working memory play a role? Memory and cognition, 40, 580-589. https://doi.org/10.3758/s13421-015-0579-2

Kane, M. J., Hambrick, D. Z., Tuholski, S. W., Wilhelm, O., Payne, T. W., and Engle, R. W. (2004). The generality of working memory capacity: a latent-variable approach to verbal and visuospatial memory span and reasoning. Journal of Experimental Psychology: General, 133(2), 189-217.

Kray, J., and Fehér, B. (2017). Age differences in the transfer and maintenance of practice-induced improvements in task switching: the impact of working-memory and inhibition demands. Frontiers in Psychology, 8, 410.

Li, C. H., He, X., Wang, Y. J., Hu, Z., and Guo, C. Y. (2017). Visual working memory capacity can be increased by training on distractor filtering efficiency. Frontiers in Psychology, 8, 196.

Luttenberger, S., Wimmer, S., y Paechter, M. (2018). Spotlight on math anxiety. Psychology Research and Behavior Management, 11, 311-322. https://doi.org/10.2147/PRBM.S141421

Mammarella, I. C., Hill, F., Devine, A., Caviola, S., y Szücs, D. (2015). Math anxiety and developmental dyscalculia: A study on working memory processes. Journal of Clinical and Experimental Neuropsychology, 37(8), 878-887. https://doi.org/10.1080/1380339 5.2015.1066759

Mavilidi, M.-F., Hoogerheide, V., y Paas, F. (2014). A Quick and Easy Strategy to Reduce Test Anxiety and Enhance Test Performance. Applied Cognitive Psychology, 28(5), 720-726. http://doi.org/10.1002/acp.3058

McAuley, A. J. (2015). The effect of gender, not math anxiety, on working memory tasks (Universidad de Nevada). http://digitalscholarship.unlv.edu/cgi/viewcontent. cgi?article $=3884 \&$ context $=$ thesesdissertations

Mutodi, P., y Ngirande, H. (2014). Exploring Mathematics Anxiety: Mathematics Students' Experiences. Mediterranean Journal of Social Sciences, 5(1), 283.

Novak, E., y Tassell, J. L. (2017). Studying preservice teacher math anxiety and mathematics performance in geometry, word, and non-word problem solving. Learning and Individual Differences, 54, 20-29. https://doi.org/10.1016/j.lindif.2017.01.005

Passolunghi, M. C., Caviola, S., De Agostini, R., Perin, C., y Mammarella, I. C. (2016). Mathematics Anxiety, Working Memory, and Mathematics Performance in 
Secondary-School Children. Frontiers in Psychology, 7. http://doi.org/10.3389/ fpsyg.2016.00042

Peng, P., Namkung, J., Barnes, M., y Sun, C. (2016). A meta-analysis of mathematics and working memory: Moderating effects of working memory domain, type of mathematics skill, and sample characteristics. Journal of Educational Psychology, 108(4), 455473. https://doi.org/10.1037/edu0000079

Pletzer, B., Wood, G., Scherndl, T., Kerschbaum, H.H., y Nuerk, H.-C. (2016). Components of Mathematics Anxiety: Factor Modeling of the MARS30-Brief. Frontiers in Psychology, 7. https://doi.org/10.3389/fpsyg.2016.00091

Rabab'h, B., y Veloo, A. (2015). Spatial Visualization as Mediating between Mathematics Learning Strategy and Mathematics Achievement among 8th Grade Students. International Education Studies, 8(5), 1-11.

Ramirez, G., Shaw, S. T., y Maloney, E. A. (2018). Math Anxiety: Past Research, Promising Interventions, and a New Interpretation Framework. Educational Psychologist, 53(3), 145-164. https://doi.org/10.1080/00461520.2018.1447384

Ramos, D. E. G., Zamora-Lugo, S. y Figueroa-Rodriguez, S. (2018). Habilidades aritméticas en estudiantes universitarios. Eduscientia, 1(1), 100-108.

Richardson, F. C., y Suinn, R. M. (1972). The mathematics anxiety rating scale: Psychometric data. Journal of Counseling Psychology, 19, 551-554. https://doi.org/10.1037/h0033456

Ruff, S. E., y Boes, S. R. (2014). The Sum of All Fears: The Effects of Math Anxiety on Math Achievement in Fifth Grade Students and the Implications for School Counselors. Georgia School Counselors Association Journal, 21(1). http://eric. ed.gov/?q=math+anxiety\&ft=on\&id=EJ1084441

Saeed, T. (2011). A Comparative Study of Working Memory in Children with Neurodevelopmental Disorders (Doctoral dissertation, National University of Ireland Maynooth).

Shi, Z., y Liu, P. (2016a). Worrying Thoughts Limit Working Memory Capacity in Math Anxiety. PLoS ONE, 11(10), 1-12. https://doi.org/10.1371/journal.pone.0165644

Shi, Z., y Liu, P. (2016b). Worrying Thoughts Limit Working Memory Capacity in Math Anxiety. PLoS ONE, 11(10), 1-12. https://doi.org/10.1371/journal.pone.0165644

Sovansky, E. E. (2013). The Relationship between State Math Anxiety and Working Memory. http://www.indigo.lib.uic.edu:8080/handle/10027/21216

Tobias, S., y Weissbrod, C. (1980). Anxiety and Mathematics: An Update. Harvard Educational Review, 50(1), 63-71.

Vallée-Tourangeau, F., Sirota, M., y Vallée-Tourangeau, G. (2016). Interactivity mitigates the impact of working memory depletion on mental arithmetic performance. Cognitive Research: Principles and Implications, 1(1), 26. https://doi.org/10.1186/ s41235-016-0027-2 
Vukovic, R. K., Kieffer, M. J., Bailey, S. P., y Harari, R. R. (2013). Mathematics anxiety in young children: Concurrent and longitudinal associations with mathematical performance. Contemporary Educational Psychology, 38(1), 1-10. https://doi.org/10.1016/j. cedpsych.2012.09.001

Walker, E. (2013). Understanding the role of metacognition and working memory in maths achievement. (Tesis doctoral no publicada). Universidad de Southampton.

Wright, E. K. C. (2017). Understanding Math anxiety in children: deciphering the contribution of math achievement, working memory, and general anxiety. (Tesis doctoral no publicada), University of South Carolina. http://search.proquest.com/ openview/9f9b1efa5ef260735d7c060eb6136001/1?pq-origsite=gscholardcbl=18750\&diss=y

Yang, H., y Yang, S. (2017). Are all interferences bad? Bilingual advantages in working memory are modulated by varying demands for controlled processing. Bilingualism: Language and Cognition, 20(1), 184-196.

Yue, Q., Martin, R. C., Fischer-Baum, S., Ramos-Nuñez, A. I., Ye, F., y Deem, M. W. (2017). Brain modularity mediates the relation between task complexity and performance. Journal of cognitive neuroscience, 29(9), 1532-1546.

Zamora Lugo, S., López Sánchez, J. D., Granados Ramos, D. E., y Romero Esquiliano, G. (2018). F109. An event-related brain potential study of the arithmetic skills in college students. Clinical Neurophysiology, 129, e107. https://doi.org/10.1016/j.clinph.2018.04.272

ISMAEL ESQUIVEL GÁMEZ

Domicilio: $\quad$ Almagro 48, Virginia, Boca del Río, Veracruz

Teléfono: $\quad$ 229-9814670 